\title{
Altered expression of glycoprotein non-metastatic melanoma protein $B$ in the distal sciatic nerve following injury
}

\author{
YANI ZHENG ${ }^{1,2^{*}}$, CHAO HUANG $^{1 *}$, XIANGQUN YANG $^{1}$ and ZHIYING ZHANG ${ }^{1}$ \\ ${ }^{1}$ Department of Anatomy, Institute of Biomedical Engineering, Second Military Medical University, Shanghai 200433; \\ ${ }^{2}$ Department of Anatomy, Fujian Health College, Fuzhou, Fujian 350101, P.R. China
}

Received September 13, 2019; Accepted March 10, 2020

DOI: $10.3892 / \mathrm{ijmm} .2020 .4559$

\begin{abstract}
Glycoprotein non-metastatic melanoma protein B (GPNMB) exerts neuroprotective effects on amyotrophic lateral sclerosis and cerebral ischemia reperfusion injury in the central nervous system. However, the expression and function of GPNMB in the peripheral nervous system, particularly following peripheral nerve injury, remains unknown. In the present study, the mRNAs and long non-coding RNAs of the distal sciatic nerve were profiled via microarray analysis at days $0,1,3,7,14,21$ and 28 following transection. The results revealed that the expression of GPNMB mRNA was similar to the proliferation tendency of distal acute denervated Schwann cells (SCs), the results of which were further validated by reverse transcription quantitative polymerase chain reaction, western blot analysis and immunohistochemistry. To investigate the function of GPNMB on SCs, recombinant human GPNMB (rhGPNMB) was added to cultured denervated SCs from the distal stumps of transected sciatic nerve. The proliferation, expression and secretion of neurotrophic factors (NTFs) and neural adhesion molecules (NAMs) were subsequently detected. The results demonstrated that GPNMB expression was increased in distal sciatic nerve following transection in vivo, while rhGPNMB promoted the proliferation of SCs as well as expression and secretion of NTFs and NAMs in vitro. Therefore, GPNMB could be a novel strategy for peripheral nerve regeneration.
\end{abstract}

Correspondence to: Dr Xiangqun Yang or Dr Zhiying Zhang, Department of Anatomy, Institute of Biomedical Engineering, Second Military Medical University, 800 Xiangyin Road, Shanghai 200433, P.R. China

E-mail: yangxq_sh@126.com

E-mail: zhiying1963@163.com

${ }^{*}$ Contributed equally

Key words: glycoprotein non-metastatic melanoma protein B, Schwann cells, sciatic nerve, peripheral nerve regeneration

\section{Introduction}

Peripheral nerve injury (PNI) is a common condition caused by trauma, burn damage or surgical intervention, and often results in permanent motor function disability and sensory perception (1). The peripheral nervous system (PNS) differs from the central nervous system (CNS) as it exhibits a certain capacity for axonal self-regeneration. However, the spontaneous regeneration of PNS is always incomplete and results in poor clinical outcomes (2). Schwann cells (SCs) have gained increasing attention in the field of peripheral nerve regeneration (PNR) owing to their vital role in the process (3). Following PNI, axons of distal impaired segments exhibit dystrophy, leading to axonal demyelination and Wallerian degeneration, while the expression of SC genes alter rapidly, switching the neuro-transmitter state to a pro-regenerative state and forming Bungner's bands (4). SCs proliferate and signal to cells to aggregate in the lesions to clear axons and myelin debris (5). They also secrete neurotrophic factors (NTFs) to prevent the apoptosis of damaged neurons and provide an enabling environment for axonal regeneration (6). However, the detailed mechanisms underlying SCs PNR are complex; they are not fully understood and require further investigation.

Glycoprotein non-metastatic melanoma protein B (GPNMB), also known as hematopoietic growth factor inducible neurokinin-1 (HGFIN), osteoactivin (rat orthologue) and DC-HIL (mouse orthologue), is widely expressed in various tissues and cells, including breast cancer cells, macrophages, melanoma cells, bladder cancer cells and osteoclasts (7). It has been demonstrated that GPNMB is involved in various biological processes, including malignant tumor cell differentiation, inflammation, regeneration, invasion and metastasis (8). GPNMB also promotes the proliferation, migration and differentiation of adult bone marrow mesenchymal stem cells and dental pulp cells $(9,10)$. Additionally, GPNMB has been reported to have neuroprotective functions in amyotrophic lateral sclerosis (11) and cerebral ischemia reperfusion injury (12), and protective effects against mutant TDP-43-induced motor neuron cell death (13) in the CNS. Recently, it has been determined that the expression levels of GPNMB in SCs harvested from injured sciatic nerves are increased in vitro (14). However, the expression of GPNMB in vivo, as well as its role following PNI, remains unknown. 
The present study investigated the expression of GPNMB in the distal sciatic nerve following transection via microarray analysis, the results of which were validated by reverse transcription-quantitative PCR (RT-qPCR), western blot analysis (WB) and immunohistochemistry (IHC). Furthermore, denervated SCs harvested from distal stumps post-sciatic nerve transection were treated with various doses of recombinant human GPNMB (rhGPNMB). Then, cell proliferation, expression and secretion of NTFs and neural adhesion molecules (NAMs) were detected in vitro. The results revealed that GPNMB promoted the proliferation of SCs, as well as the expression and secretion of NTFs and NAMs. The results indicated that GPNMB may be utilized as a novel treatment strategy for PNR.

\section{Materials and methods}

Animals and tissue preparation. Adult male Sprague-Dawley (SD) rats were obtained from the Animal Center of Second Military Medical University and all animal experiments were approved and supervised by the Animal Care and Use Committee of the Second Military Medical University (permit no. SYXK-2002-042). All rats were kept in cages under controlled conditions at $22^{\circ} \mathrm{C}$ with ad libitum access to food and water and a 12-12 $\mathrm{h}$ day/night cycle.

A total of 84 rats (weight, 180-200 g) were randomly divided into seven groups of 12 rats each. Following anesthesia by intraperitoneal injection of pentobarbital sodium $(40 \mathrm{mg} / \mathrm{kg})$, the bilateral sciatic nerves were exposed $2 \mathrm{~mm}$ from distal ischial tuberosity and transected with fine scissors, after which the incision sites were closed. No rats died following sciatic nerve transection. Then, the distal injured sciatic nerves of each rat were obtained in $5 \mathrm{~min}$ at day $0,1,3,7,14,21$ and 28 post-transection. After distal sciatic nerves were obtained, rats remained under anesthesia and were immediately sacrificed by cervical dislocation. Following confirmation of an absence of respiration, the rats were collected and handed over to the Animal Center of Second Military Medical University for disposal.

Microarray analysis. Microarray experiments $(n=3)$ were performed by OE Biotech Co., Ltd. Total RNA was extracted using TRIzol ${ }^{\circledR}$ (Invitrogen; Thermo Fisher Scientific, Inc.) and quantified using NanoDrop ND-2000 (Thermo Fisher Scientific, Inc.). RNA integrity was assessed using the Agilent Bioanalyzer 2100 (Agilent Technologies, Inc.). Sample labeling (Quick Amp Labeling kit; cat. no. 5190-2305), microarray hybridization (Agilent Gene Expression Hybridization kit; cat. no. 5188-5242) and washing (Gene Expression Wash Buffer; cat. no. 5188-5325 and 5188-5326; all Agilent Technologies, Inc.) were performed based on the manufacturer's standard protocols. Total RNA was transcribed to double-stranded cDNA, then synthesized into cRNA and labeled with Cyanine-3-CTP. Labeled cRNAs were subsequently hybridized onto the $8 \times 60 \mathrm{~K}$ Agilent Rat long non-coding RNA (lncRNA) Array. Following washing, the arrays were scanned using the Agilent Scanner G2505C (Agilent Technologies, Inc.). Feature Extraction software (v.10.7.1.1; Agilent Technologies, Inc.) was used to analyze array images to obtain raw data. Genespring software (v.13.1; Agilent Technologies, Inc.) was utilized to conclude basic analysis using raw data.
Search for specific $m R N A$ profiles. Short time-series expression miner (STEM) analysis (15) was performed to obtain LncRNA and mRNA expression profiles, which were subsequently compared for significance and false discovery rate. Differentially expressed genes at certain time points were obtained via screening. Using the mRNAs with significant expression levels, hierarchical clustering to determine the distinguishable gene expression patterns among samples, Gene ontology (GO) $(16,17)$ and Kyoto Encyclopedia of Genes and Genomes (KEGG) (18) enrichment analyses were conducted to determine the roles of these differentially expressed mRNAs.

Culture of denervated SCs from distal stumps of resected sciatic nerve. Denervated SCs were harvested as previously described (19). A total of 4 weeks after sciatic nerve transection, distal stumps were harvested and washed with PBS, after which the epineurium and perineurium were stripped and the tissue was sliced into $1 \times 1 \times 1 \mathrm{~mm}$ fragments. The fragments were then cultured in DMEM Nutrient Mixture F-12 (Gibco; Thermo Fisher Scientific, Inc.) supplemented with 5\% fetal bovine serum (FBS; Gibco; Thermo Fisher Scientific, Inc.). After 7 days of preparation, nerve segments were seeded in poly-L-ornithine/Laminin (Gibco; Thermo Fisher Scientific, Inc.) coated 6- or 24-well plates and cultured in culture medium consisting of DMEM/F12, 10\% FBS, $1 \%$ Glutamine, $100 \mathrm{U} / \mathrm{ml}$ penicillin and $100 \mu \mathrm{g} / \mathrm{ml}$ streptomycin (Gibco; Thermo Fisher Scientific, Inc.) and $5 \mu \mathrm{M}$ forskolin (Sigma-Aldrich; Merck $\mathrm{KGaA}$ ). After $48 \mathrm{~h}$ incubation, SCs were released from the nerve segments. The medium was replaced with culture medium supplemented with 2 M cytarabine (Sigma-Aldrich; Merck KGaA) for purification. After $72 \mathrm{~h}$, the medium with cytarabine was replaced with culture medium. When cells grew to $90 \%$ confluence, they were passaged. Cells from passage 4 were used for all experiments.

rhGPNMB treatment. rhGPNMB (R\&D Systems, Inc.), which included the sequences of human GPNMB from Lysine 23 to Asparagine 486, was dissolved in PBS. Passage 4 SCs were divided into four groups and treated with 0, 10, 50 and $100 \mathrm{nM}$ rhGPNMB, respectively. The $0 \mathrm{nM}$ rhGPNMB-treated group served as the control. SCs were treated for $48 \mathrm{~h}$, after which they were examined.

SC proliferation assay. SC proliferation was determined using a Cell Counting Kit 8 (CCK8; Dojindo Molecular Technologies, Inc.) assay. rhGPNMB treated SCs were seeded into 96-well plates at 3,000 cells/well and incubated for $24 \mathrm{~h}$ in a humidified atmosphere with $5 \% \mathrm{CO}_{2}$. Cells were then incubated with $10 \mu \mathrm{l} \mathrm{CCK} 8$ solution per well at $37^{\circ} \mathrm{C}$ for $1 \mathrm{~h}$, after which the absorbance was measured using a microplate reader (BioTek Instruments, Inc.) at $450 \mathrm{~nm}$. Results were presented as mean optical density values $(n=6)$.

$R T-q P C R$. Total RNA $(\mathrm{n}=6)$ was extracted using TRIzol ${ }^{\circledR}$ (Invitrogen; Thermo Fisher Scientific, Inc.) and concentration was detected using a microplate reader (BioTek Instruments, Inc.). cDNA was subsequently synthesized using the PrimeScript ${ }^{\mathrm{TM}}$ RT Reagent kit (Takara Bio, Inc.) in accordance with the manufacturer's protocol. The expression of mRNA was determined by SYBR-Green I-based qPCR using the 
Table I. Sequences of primers used for RT-qPCR analysis.

\begin{tabular}{lll}
\hline Target & \multicolumn{1}{c}{ Forward (5'-3') } & \multicolumn{1}{c}{ Reverse (5'-3') } \\
\hline GPNMB & ATGTCCTCATTCACGATCC & TGAAGGTTCCATTGAGCAC \\
NGF & CATCACTGTGGACCCCAAACTGT & GTCCGTGGCTGTGGTCTTATCTC \\
BDNF & TTGATGAGACCGGGTTCCCT & GTCCGTGGACGTTTGCTTCTT \\
NT-3 & GCGATGCTACCGCAAAGAAC & ATGGCGTCTCCCACACTACC \\
NCAM & CAAGTCCCTAGACTGGAACGC & CCTTGGATTTCCTTGCTGGT \\
N-cadherin & CCAAATCCTAAAATCATTCGCC & CACAGGGTCTATTTTCAGCCAGT \\
GAPDH & GCCATCAACGACCCCTTCAT & TTCACACCCATCACAAACA
\end{tabular}

GPNMB, glycoprotein non-metastatic melanoma protein B; NGF, nerve growth factor; BDNF, brain-derived neurotrophic factor; NT-3, neurotrophin 3; NCAM, neural cell adhesion molecule.

Bio-Rad 3000 Quantitative Real-time PCR System (Bio-Rad Laboratories, Inc.). The primers used for PCR are presented in Table I. The efficiency and specificity values of each primer set were confirmed by comparing the standard curves of the threshold cycle values to the RNA serial dilutions and melting curve evaluations. GAPDH was used as an internal control to normalize gene expression levels and the relative expression of target mRNA was calculated using the $2^{-\Delta \Delta \mathrm{Cq}}$ method (20).

$W B$. Distal sciatic nerves $(\mathrm{n}=6)$ were harvested at day $0,1,3,7$, 14,21 and 28 post-transection and SCs that were treated with rhGPNMB for $48 \mathrm{~h}$ were washed with PBS, lysed using RIPA assay buffer (cat. no. G2002-100; Service Biotechnology Co., Ltd.) and centrifuged at $4^{\circ} \mathrm{C}$ at $12,000 \mathrm{x}$ g for $10 \mathrm{~min}$ to harvest the supernatant. Protein concentration was then detected using a Bicinchoninic Acid kit (Shanghai Yeasen Biotechnology Co., Ltd.). The supernatant was boiled at $100^{\circ} \mathrm{C}$ and centrifuged at $1,000 \mathrm{x}$ g for $5 \mathrm{~min}$ for later use. Following preparation of the separation gel, SDS-PAGE was performed. An equal quantity $(10 \mu \mathrm{g} / \mathrm{lane})$ of extracted protein was resolved on a (10-15\%) polyacrylamide gradient. Proteins were then transferred to a nitrocellulose membrane. The membrane was blocked in 5\% skimmed milk/PBS for $1 \mathrm{~h}$ at $4^{\circ} \mathrm{C}$ and then incubated overnight at $4^{\circ} \mathrm{C}$ with the following primary antibodies: Rabbit polyclonal anti-GPNMB (cat. no. GXP212342; 1:2,000; GenXspan, Inc.), rabbit monoclonal anti-nerve growth factor (NGF; cat. no. ab6199; 1:2,000; Abcam), rabbit monoclonal anti-brain-derived neurotrophic factor (BDNF; cat. no. ab108319; 1:2,000; Abcam), rabbit polyclonal anti-neurotrophin 3 (NT-3; cat. no. ab16640; 1:2,000; Abcam), mouse monoclonal anti-neural cell adhesion molecule (NCAM; cat. no. ab9018; 1:2,000; Abcam), mouse monoclonal anti-neural cadherin (N-cadherin; cat. no. ab98952; 1:2,000; Abcam) and GAPDH (cat. no. WB2197; 1:2,000; Biotech Well Co., Ltd.). The sample was then incubated at $4^{\circ} \mathrm{C}$ for $1 \mathrm{~h}$ with horseradish peroxidase (HRP)-labeled secondary antibodies which were diluted in $0.01 \mathrm{M}$ TBST; goat anti-rabbit secondary antibody (cat. no. 111-035-003; 1:5,000) and goat anti-mouse secondary antibody (cat. no. 115-035-003; 1:5,000, both Jackson ImmunoResearch Laboratories, Inc.). Subsequently, the membrane was incubated with the ECL hypersensitive chemiluminescence kit (cat. no. WB0164; Biotech Well Co., Ltd.) and chemiluminescence was detected following exposure to
X-rays. The bands were quantified by measuring the band intensity of each group and normalizing it to GAPDH using Odyssey v.1.2 software (LI-COR Biosciences).

IHC. An IHC kit (Wuhan Boster Biological Technology, Ltd.) was used to perform IHC in accordance with the manufacturer's protocol. Distal segments of transected sciatic nerve were fixed in $4 \%$ paraformaldehyde at $4^{\circ} \mathrm{C}$ for $24 \mathrm{~h}$, dehydrated, at room temperature using a gradient alcohol series $(75 \%$ for $2 \mathrm{~h}, 85 \%$ for $1.5 \mathrm{~h}, 95 \%$ for $1 \mathrm{~h}$, and $100 \%$ for $30 \mathrm{~min}$ ), vitrified by dimethylbenzene for 2 min, embedded in paraffin, and sectioned. Sections were subsequently baked at $60^{\circ} \mathrm{C}$ for $1 \mathrm{~h}$, deparaffinated at room temperature by dimethylbenzene ( 2 times for $15 \mathrm{~min}$ each), treated in a gradient alcohol series (100\% for $5 \mathrm{~min}, 95 \%$ for $5 \mathrm{~min}, 85 \%$ for $5 \mathrm{~min}$ and $75 \%$ for $5 \mathrm{~min}$ ), rehydrated, subjected to antigen retrieval by heating sections in $0.01 \mathrm{M}$ sodium citrate buffer (pH 6.0) for $15 \mathrm{~min}$ at $96-98^{\circ} \mathrm{C}$ and cooled to room temperature. Following peroxidase blocking with $3 \%$ hydrogen peroxide at room temperature for $10 \mathrm{~min}$, samples were further blocked with blocking solution containing 10\% goat serum (cat. no. AR1009; Wuhan Boster Biological Technology, Ltd.) at $37^{\circ} \mathrm{C}$ for $30 \mathrm{~min}$. Sections were then incubated with rabbit polyclonal anti-GPNMB primary antibody (1:50; GenXspan, Inc.) at $4^{\circ} \mathrm{C}$ overnight followed by incubation at room temperature for $30 \mathrm{~min}$. Following washing with $0.01 \mathrm{M}$ PBS, sections were incubated with biotin-labeled anti-rabbit IgG secondary antibody (1:200; cat. no. SA1052; Wuhan Boster Biological Technology, Ltd.) for $1 \mathrm{~h}$ at $37^{\circ} \mathrm{C}$. Then, $0.01 \%$ DAB solution (Wuhan Boster Biological Technology, Ltd.) was added according to the manufacturer's protocol, sections were rinsed with distilled water and sealed with neutral resin. Images were captured under a microscope (magnification, x200; Leica Microsystems $\mathrm{GmbH})(\mathrm{n}=3)$.

ELISA. ELISA kits was used to measure levels of NGF (cat. no. ER0143), BDNF (cat. no. ER0102), NT-3 (cat. no. ER0144; all Biotech WellCo.,Ltd.), NCAM (cat.no.EK1562) and N-cadherin (cat. no. EK1156, both Wuhan Boster Biological Technology, Ltd.) in culture medium following the manufacturer's protocol. SCs were seeded into 6 -well plates at $5 \times 10^{5}$ cells/well and cultured in serum-free medium. After $48 \mathrm{~h}$ induction, supernatants were collected and used to measure NGF, BDNF, NT-3, NCAM and N-cadherin levels secreted by SCs $(n=8)$. 

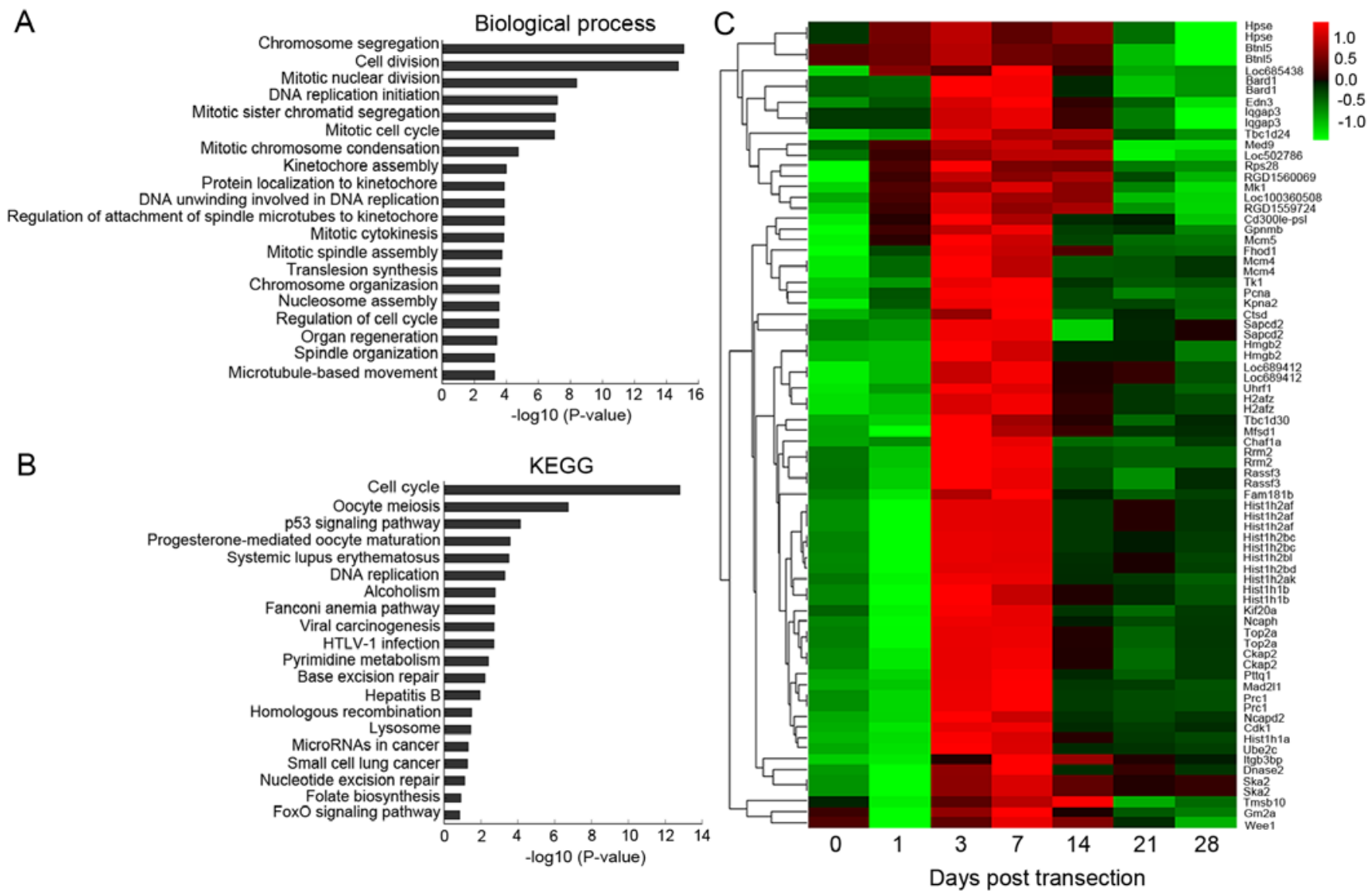

Figure 1. Bioinformatics analyses of microarray data from the distal sciatic nerve at days 0, 1, 3, 7, 14, 21 and 28 following transection. (A) Gene Ontology and (B) Kyoto Encyclopedia of Genes and Genomes pathway analyses revealed that profile 41 may be associated with cell proliferation after transection. (C) Heatmap and cluster dendogram demonstrated 76 mRNAs with significantly differential expressions at the 7 indicated time points post injury. The color scale on the right-hand side illustrates the relative expression level of mRNAs across all samples: Red denotes expression $>0$ and green denotes expression $<0$.

Statistical analysis. All the aforementioned tests were performed at least in triplicate and were repeated at least 3 times. The results were expressed as the mean \pm standard deviation and analyzed using SPSS v.21.0 software (IBM Corp.). Figures were generated using Prism v.6.0 software (GraphPad Software, Inc.). A one way analysis of variance followed by Tukey's post hoc test was used for multiple comparisons. $\mathrm{P}<0.05$ was considered to indicate a statistically significant difference.

\section{Results}

Bioinformatics analyses of microarray data. Microarray data were divided into 50 profiles to summarize the expression of mRNAs and long non-coding RNAs (lncRNAs) via STEM analysis. The expression tendency of profile 41, which increased, peaked and then decreased was similar to the proliferation tendency of distal acute denervated SCs. Considering the vital roles of SCs in PNR, profile 41 was selected for further analyses.

Profile 41 consisted of 76 mRNAs and 282 lncRNAs, and was selected for bioinformatics analyses. The results of GO analysis (Table SI) revealed that profile 41 was associated with cell division, mitotic nuclear division and DNA replication initiation (Fig. 1A). KEGG analysis (Table SII) confirmed the results of GO analysis, demonstrating that profile 41 was associated with the cell cycle, the p53 signaling pathway and DNA replication (Fig. 1B). The results of GO and KEGG analyses indicated that profile 41 may be involved in cell proliferation following transection. In addition, hierarchical clustering analysis was performed to reveal systematic variations in the 76 markedly altered mRNA expression profiles of profile 41 (Fig. 1C). The expression level of GPNMB, the most significantly altered mRNA, increased from day 1 , peaked at day 7 , which was $\sim 48$-fold higher compared with that of day 0 , and then gradually decreased from day 14 .

Altered expression of GPNMB in distal sciatic nerves post-transection. GPNMB, the most significantly altered mRNA, was selected for analysis via RT-qPCR, WB and IHC in distal stumps at certain time points post-transection. The results revealed that the expression of GPNMB increased at day $1(3.87 \pm 0.43$-fold increase compared with that of day $0 ; \mathrm{P}<0.05)$, rapidly rose from day 3 , peaked at day 7 (48.63 \pm 3.71 -fold increase compared with that of day $0, \mathrm{P}<0.05$ ) and gradually decreased from day 14 to day 28 (Fig. 2A). The alteration tendency of GPNMB at the transcriptional level was consistent with microarray data. Furthermore, similar results in GPNMB protein expression were obtained by WB (Fig. 2B and C). The results revealed that the expression of GPNMB rose at day 1 and 3 post injury, significantly increased from day 7, peaked at day 21 and then gradually decreased at 

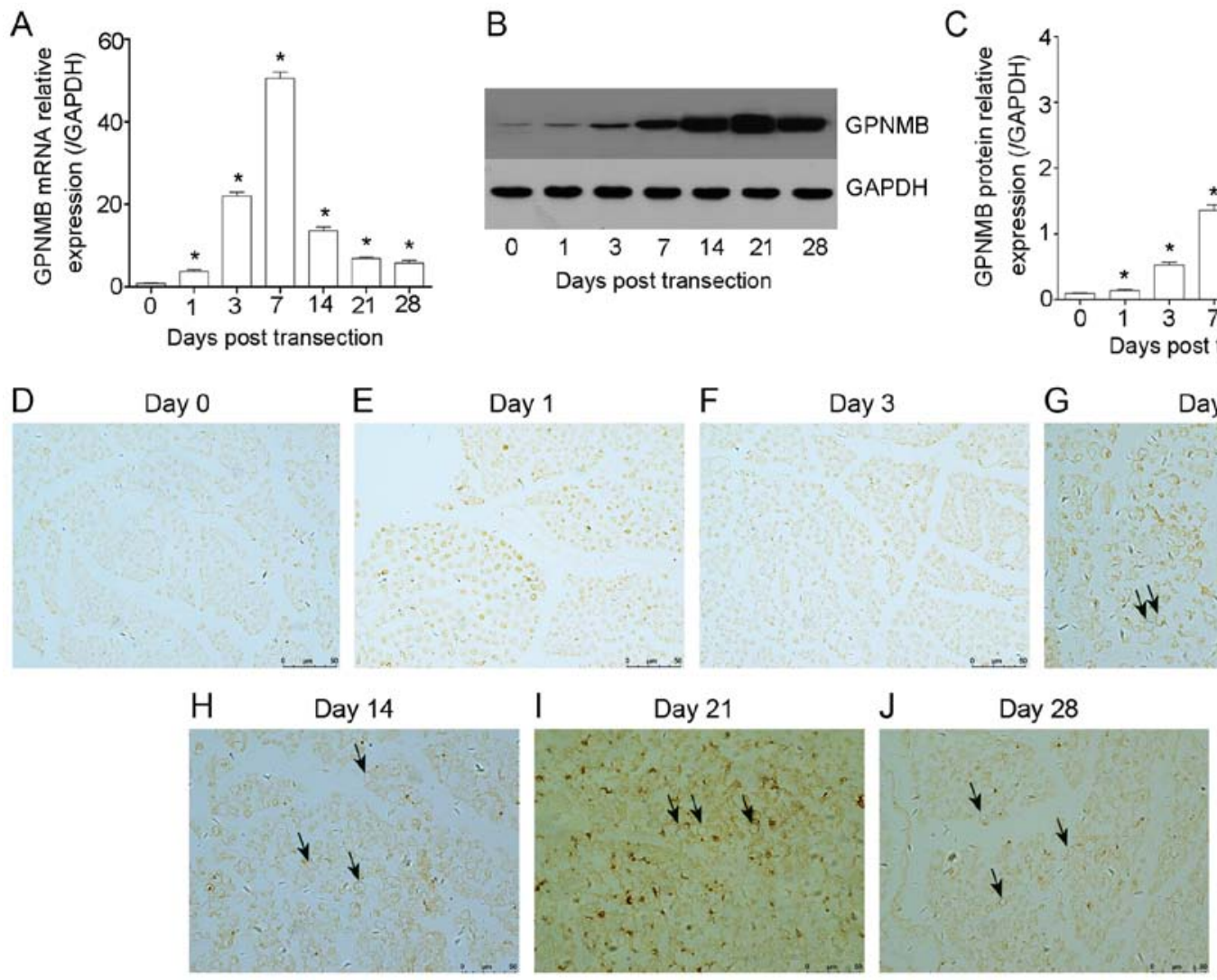

Figure 2. Validation of GPNMB in distal sciatic nerve at serial time points post-transection. (A) Reverse transcription-quantitative $\mathrm{PCR}(\mathrm{n}=6)$, (B and C) western blot analysis $(n=6)$ and (D-J) immunohistochemistry $(n=3)$ were performed to detect the expression of GPNMB. The black arrow denotes GPNMB expressed around nerve fibers. "P<0.05 vs. day 0 . Analyses were performed using one-way analysis of variance and Tukey's post hoc test. GPNMB, glycoprotein non-metastatic melanoma protein $\mathrm{B}$.

day 28. Although the time point at which GPNMB reached its peak varied following RT-qPCR and WB, the results were concomitant with the microarray data.

IHC was performed to clarify the expression and localization of GPNMB in transverse (Fig. 2D-J) and longitudinal (Fig. S1) sections following transection. The results revealed that the expression of GPNMB was consistent with WB. Additionally, it was demonstrated that GPNMB was expressed around the nerve fibers, indicating that GPNMB may be expressed in SCs (Fig. 2).

These results demonstrated that the expression of GPNMB was altered in the distal sciatic nerves post-transection.

GPNMB promotes the proliferation of SCs. Following exposure to various doses of rhGPNMB for $48 \mathrm{~h}$, the proliferation of SCs was detected using CCK-8 (Fig. 3). The results revealed that SCs proliferated at an increased rate compared with the control when cultured with rhGPNMB $(\mathrm{P}<0.05)$. Additionally, SCs treated with $50 \mathrm{nM}$ rhGPNMB grew more rapidly compared with the other 2 groups (10 and $100 \mathrm{nM})$, indicating that the proliferation of SCs increased in a dose-dependent manner $(\mathrm{P}<0.05)$.

GPNMB accelerates the expression and secretion of NTFs. NTFs, including NGF, BDNF and NT-3, serve significant roles in nerve regeneration. Following treatment with various doses of rhGPNMB, RT-qPCR and WB were performed to measure the expression of NGF, BDNF and NT-3 in SCs.

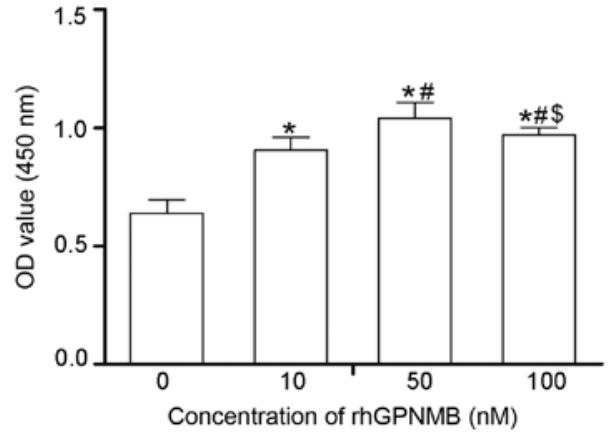

Figure 3. Cell proliferation of SCs $(n=6)$. Following treatment with rhGPNMB, SC proliferation gradually rose in a dose-dependent manner. ${ }^{*} \mathrm{P}<0.05$ vs. 0 nM. ${ }^{\#} \mathrm{P}<0.05$ vs. 10 nM. ${ }^{\$} \mathrm{P}<0.05$ vs. $50 \mathrm{nM}$. Analyses were performed using one-way analysis of variance and Tukey's post hoc test. SCs, Schwann cells; rhGPNMB, recombinant human glycoprotein non-metastatic melanoma protein $\mathrm{B}$.

The results determined that SCs treated with rhGPNMB expressed increased levels of NGF, BDNF and NT-3 compared with the control ( $\mathrm{P}<0.05$; Fig. 4A). Furthermore, the expression of NGF was 4-5-fold higher in the $50 \mathrm{nM}$ rhGPNMB treated group compared with the control. It was also higher compared with the other two rhGPNMB treated groups $(\mathrm{P}<0.05)$. In addition, the expression levels of BDNF and NT-3 were significantly increased in the $50 \mathrm{nM}$ rhGPNMB-treated group compared with the other three groups $(\mathrm{P}<0.05)$. These results indicated that the expression 


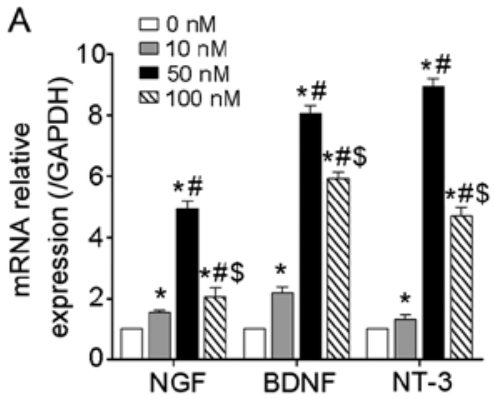

D

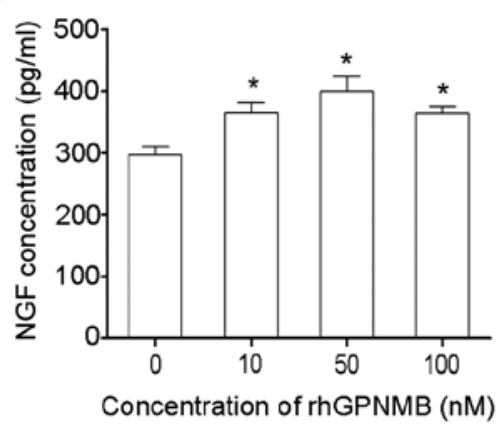

B

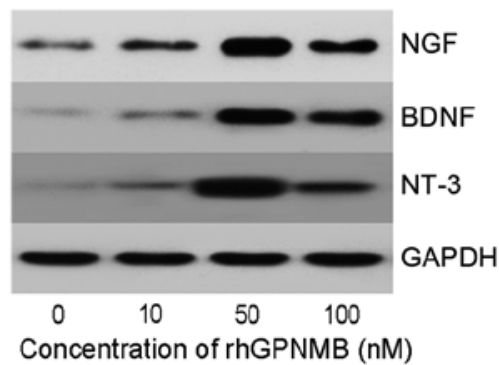

$\mathrm{E}$

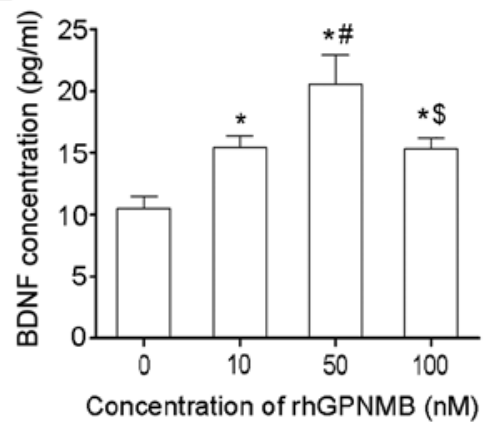

C

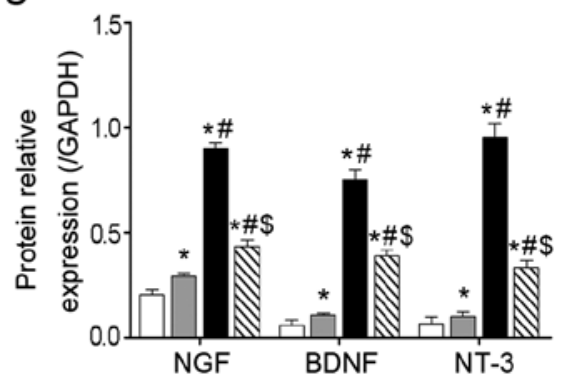

$\mathrm{F}$

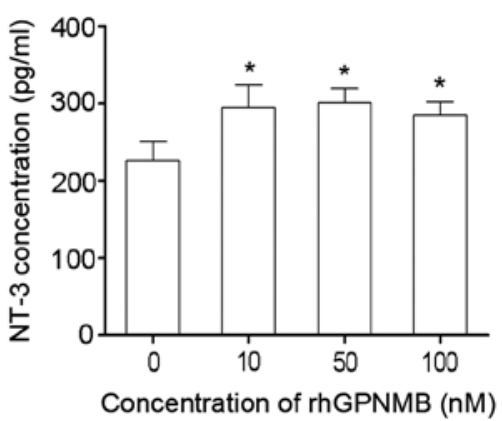

Figure 4. Expression and secretion of NGF, BDNF and NT-3 in SCs following treatment with rhGPNMB. (A) Reverse transcription-quantitative PCR (n=6), (B and C) western blot analysis $(n=6)$ and $(\mathrm{D}-\mathrm{F})$ ELISA $(\mathrm{n}=8)$ were performed to detect the expression levels of NGF, BDNF and NT-3 in Schwann cells. ${ }^{*} \mathrm{P}<0.05$ vs. $0 \mathrm{nM},{ }^{\#} \mathrm{P}<0.05$ vs. $10 \mathrm{nM}$. ${ }^{\$} \mathrm{P}<0.05$ vs. $50 \mathrm{nM}$. Analyses were performed using one-way analysis of variance and Tukey's post hoc test. NGF, nerve growth factor; BDNF, brain-derived neurotrophic factor; NT-3, neurotrophin 3; rhGPNMB, recombinant human glycoprotein non-metastatic melanoma protein B.

of NGF, BDNF and NT-3 at the transcriptional level increased significantly in a dose-dependent manner in SCs following treatment with rhGPNMB. Similar results were also obtained via WB (Fig. 4B and C). The expression of NGF, BDNF and NT-3 at the protein level was also significantly increased in the $50 \mathrm{nM}$ rhGPNMB-treated group compared with the other three groups $(\mathrm{P}<0.05)$. Additionally, the expression of NGF, BDNF and NT-3 at the protein level was increased in the 10 and $100 \mathrm{nM}$ rhGPNMB-treated groups compared with the control $(\mathrm{P}<0.05)$.

ELISAs were performed to measure the secretion levels of NGF, BDNF and NT-3 in SCs. The results revealed that SCs secreted an increased level of NGF, BDNF and NT-3 compared with the control following treatment with rhGPNMB $(\mathrm{P}<0.05$; Fig. 4D-F). However, there were no significant differences in the secretions of NGF and NT-3 among the three rhGPNMB-treated groups ( $\mathrm{P}>0.05$; Fig. 4D and F). The secretion of BDNF was increased in the $50 \mathrm{nM}$ rhGPNMB treated group compared with the other rhGPNMB treated groups $(\mathrm{P}<0.05$; Fig. 4E).

The aforementioned results indicated that GPNMB accelerated the expression and secretion of NGF, BDNF and NT-3 in SCs.

GPNMB triggers the expression and secretion of NAMs. The expression and secretion of certain NAMs, including NCAM and $\mathrm{N}$-cadherin, were also detected by RT-qPCR, WB and ELISA. These NAMs were selected as they can promote SC migration during the nerve regeneration processes (21). The results revealed that the SCs expressed increased levels of NCAM and N-cadherin in rhGPNMB-treated groups compared with the control group at the transcriptional level
$(\mathrm{P}<0.05$; Fig. 5A). The expression of NCAM in the $100 \mathrm{nM}$ rhGPNMB-treated group was increased compared with the other two rhGPNMB-treated groups $(\mathrm{P}<0.05)$, indicating that its expression increased in a dose-dependent manner. However, there was no significant difference in the expression of N-cadherin between the 10 and $50 \mathrm{nM}$ rhGPNMB treated groups $(\mathrm{P}>0.05)$. The results of $\mathrm{WB}$ were consistent with those of RT-qPCR (Fig. 5B and C). It was revealed that the expression of NCAM at the protein level increased in a dose-dependent manner when SCs were treated with rhGPNMB. Additionally, SCs expressed increased levels of $\mathrm{N}$-cadherin in rhGPNMB treated groups compared with the control. However, this was not dose-dependent.

Using ELISAs, the present study obtained similar results regarding the secretion of NCAM and N-cadherin in SCs following treatment with rhGPNMB (Fig. 5D and E). It was also revealed that SCs secreted increased levels of NCAM and $\mathrm{N}$-cadherin when compared with the control $(\mathrm{P}<0.05)$. However, there were no significant differences in the secretion of NCAM and N-cadherin among the three rhGPNMB-treated groups $(\mathrm{P}>0.05)$.

The results of the RT-qPCR, WB and ELISA analyses revealed that GPNMB triggered the expression and secretion of NCAM and N-cadherin in SCs.

\section{Discussion}

GPNMB, first cloned and described as a regulator protein of tumor growth (22), is ubiquitously expressed in the CNS (8) and exerts protective effects in amyotrophic lateral sclerosis by regulating the amelioration of skeletal muscle lesions, motor neuron cell death and PI3K/Akt and MEK/ERK pathway 


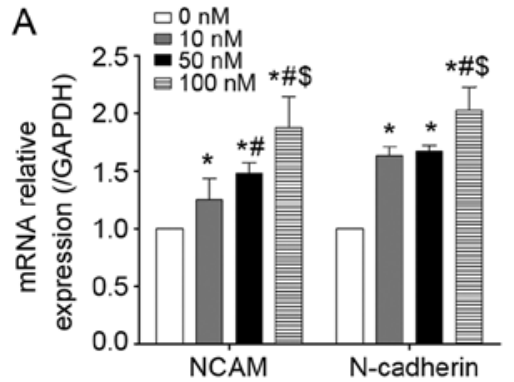

B

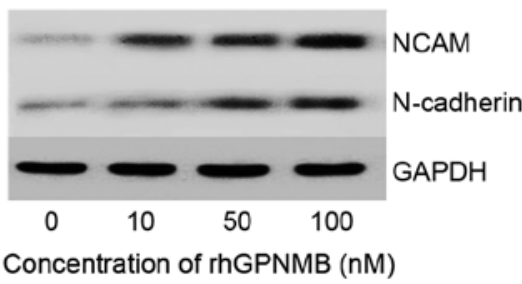

C

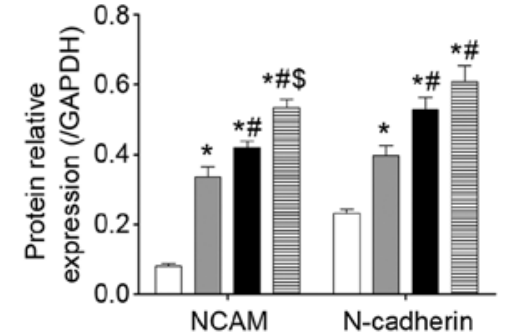

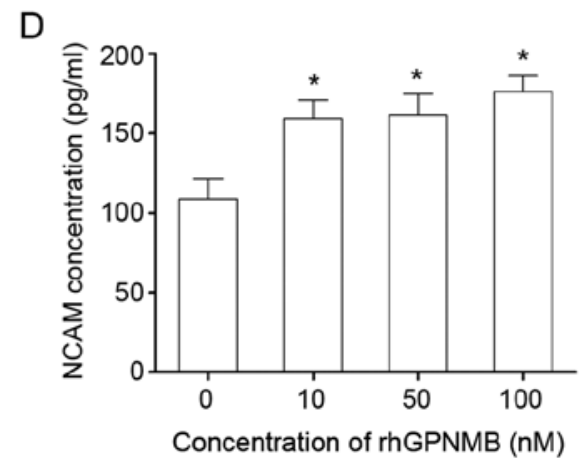

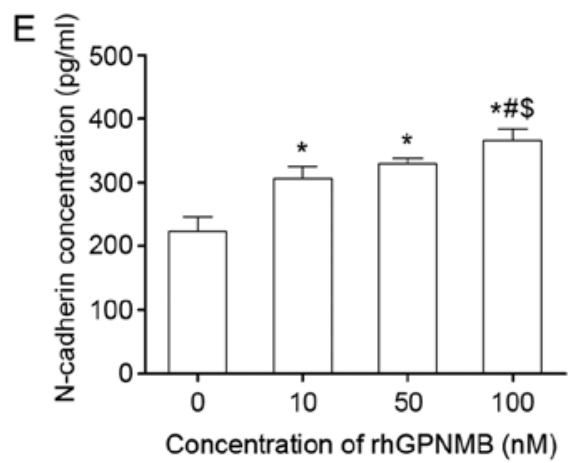

Figure 5. Expression and secretion of NCAM and N-cadherin in Schwann cells following treatment with rhGPNMB. (A) Reverse transcription-quantitative PCR $(n=6),(B$ and $C)$ western blot analysis $(n=6)$ and $(D$ and E) ELISA $(n=8)$ were performed to detect the expression of NCAM and N-cadherin. *P<0.05 vs. 0 nM. ${ }^{\# P}<0.05$ vs. 10 nM. ${ }^{\$} \mathrm{P}<0.05$ vs. $50 \mathrm{nM}$. Analyses were performed using one-way analysis of variance and Tukey's post hoc test. NCAM, neural cell adhesion molecule; N-cadherin, N-cadherin; rhGPNMB, recombinant human glycoprotein non-metastatic melanoma protein B.

activation $(11,13,23,24)$. It also has been revealed that GPNMB exerts neuroprotective effects against cerebral ischemia reperfusion injury via ERK1/2 and Akt pathways (12). Recently, it has been reported that the expression of GPNMB in SCs harvested from injured sciatic nerve increases in vitro (14). However, no reports have determined the expression of GPNMB in vivo or have assessed the associated importance of PNS injuries and diseases.

The present study used a sciatic nerve transection model to investigate gene expression profiles in distal segments via microarray analysis. The results of the STEM analysis revealed that the expression of profile 41 , which consisted of 76 mRNAs and 282 lncRNAs, increased, peaked and then gradually decreased at serial time points post-injury. The results also demonstrated the altered expression of GPNMB in vivo following injury, which was different to previous research regarding GPNMB expression in vitro (14). These results may serve an important role in the study of PNR.

PNR is a complex biological process that involves interactions among multiple cells, NTFs and extracellular matrix proteins (25). During PNR, SCs shed their myelin sheaths, dedifferentiate to a progenitor/stem cell-like state, proliferate, migrate and produce a favorable environment for axonal regeneration by helping to clear myelin debris and by forming cellular conduits for the growth and pathfinding of axons (26). In the present study, the results of the STEM analysis revealed that the expression of profile 41 was similar to that of the proliferation tendency of distal acute denervated SCs (27). Profile 41 was therefore selected for further bioinformatics analyses.

The results of the present study revealed that profile 41 was associated with cell division, mitotic nuclear division, DNA replication initiation, cell cycle, the p53 signaling pathway and DNA replication. In addition, GPNMB was the most significantly varied mRNA among the 76 included in profile 41 . These results were consistent with previous studies, which revealed that the level of cellular p53 is associated with the activity of GPNMB, also termed HGFIN, and the cell cycle, and that the p53 signaling pathway is involved in the regulation of GPNMB expression and activity $(28,29)$. Therefore, it was hypothesized that GPNMB may be involved in cell proliferation following PNI from bioinformatics analyses. The results of the present study also revealed that GPNMB promoted denervated SC proliferation in vitro, which confirmed these hypotheses.

GPNMB was selected for validation via RT-qPCR, WB and IHC. The results indicated that the altered expression of GPNMB at the transcriptional and protein levels were in concordance with the microarray analysis. Furthermore, transverse section IHC analysis revealed that GPNMB was expressed around nerve fibers, indicating that GPNMB may be expressed in SCs. These results were consistent with those of a previous study, in which the increased expression of GPNMB in SCs following injury was observed in vitro (14). However, the time point at which GPNMB peaked in WB was different to that observed following microarray analysis and RT-qPCR. The present study hypothesized that this may due to protein translation from mRNA and post-translational modification.

The microenvironment, consisting of NTFs and NAMs, has been proven to promote nerve regeneration in vitro and in vivo and serve neuroregenerative and neuroprotective roles (30). Recently, it has been demonstrated that NGF promotes the regeneration and functional recovery of impaired peripheral nerves (31). Furthermore, it has also been confirmed that BDNF (32), NT-3 (33) and N-cadherin (34) serve a vital role 
in promoting nerve regeneration after injury. In the present study, denervated SCs were harvested from injured nerves, as described in our previous study (19) and were identified to exhibit a long bipolar or tripolar elongated shape with a small oval-shaped nucleus and expressed protein S100- $\beta$ in the membrane of the nucleus and cytoplasm. The results of the present study revealed that denervated SCs expressed and secreted increased levels of NGF, BDNF, NT-3, NCAM and $\mathrm{N}$-cadherin following rhGPNMB treatment in vitro, indicating that GPNMB may promote PNR.

The expression profile of GPNMB observed in the present study was consistent with other study by proteomics published recently (14). The altered expression tendency of GPNMB, which increased, peaked, and then decreased was also similar to the proliferation of distal acute denervated SCs (27). In addition, the results revealed that GPNMB promoted the proliferation of denervated SCs as well as the expression and secretion of NTFs and NAMs in vitro. However, whether GPNMB promotes the proliferation and secretion of SCs in vivo remains unknown. Furthermore, there may be bias and statistical error in the study due to the small sample size. Therefore, future studies should assess the functions of GPNMB in vivo, particularly on the proliferation and secretion of SCs, and determine the underlying mechanisms involved in PNR.

The results of the present study revealed that GPNMB was expressed and altered in the distal sciatic nerve following transection in vivo and that GPNMB also promoted the proliferation of denervated SCs as well as the expression and secretion of NTFs and NAMs in vitro.

In conclusion, the present study demonstrated the altered expression of GPNMB in distal sciatic nerve following transection in vivo. It was also demonstrated that GPNMB promoted the proliferation of denervated SCs as well as the expression and secretion of NTFs and NAMs in vitro. The results revealed that GPNMB may be used as a novel strategy for PNR.

\section{Acknowledgements}

The authors would like to thank Professor Fang Liu and Professor Haiyan Lin (Department of Anatomy, Second Military Medical University, Shanghai, China) for helpful suggestions and technical assistance.

\section{Funding}

This study was supported by the Funds of the National Natural Science Foundation of China (grant no. 81271396).

\section{Availability of data and materials}

The datasets used and/or analyzed during the current study are available from the corresponding author on reasonable request.

\section{Authors' contributions}

$\mathrm{ZZ}$ and $\mathrm{XY}$ designed the study. $\mathrm{YZ}$ and $\mathrm{CH}$ performed the experiments. $\mathrm{YZ}$ and $\mathrm{CH}$ analyzed data. ZZ, XY, YZ and $\mathrm{CH}$ wrote the manuscript. All authors read and approved the final manuscript.

\section{Ethics approval and consent to participate}

All animal experiments were approved and supervised by the Animal Care and Use Committee of the Second Military Medical University (permit no. SYXK-2002-042).

\section{Patient consent for publication}

Not applicable.

\section{Competing interests}

The authors declare that they have no competing interests.

\section{References}

1. Ray WZ and Mackinnon SE: Management of nerve gaps: Autografts, allografts, nerve transfers, and end-to-side neurorrhaphy. Exp Neurol 223: 77-85, 2010.

2. Du J, Liu J, Yao S, Mao H, Peng J, Sun X, Cao Z, Yang Y, Xiao B, Wang Y, et al: Prompt peripheral nerve regeneration induced by a hierarchically aligned fibrin nanofiber hydrogel. Acta Biomater 55: 296-309, 2017.

3. Scheib J and Höke A: Advances in peripheral nerve regeneration. Nat Rev Neurol 9: 668-676, 2013.

4. Allodi I, Udina E and Navarro X: Specificity of peripheral nerve regeneration: Interactions at the axon level. Prog Neurobiol 98: 16-37, 2012.

5. Vargas ME and Barres BA: Why is Wallerian degeneration in the CNS so slow? Annu Rev Neurosci 30: 153-179, 2007.

6. Han B, Zhao JY, Wang WT, Li ZW, He AP and Song XY: Cdc42 promotes schwann cell proliferation and migration through $W n t / \beta$-catenin and p38 MAPK signaling pathway after sciatic nerve injury. Neurochem Res 42: 1317-1324, 2017.

7. Taya M and Hammes SR: Glycoprotein non-metastatic melanoma protein B (GPNMB) and cancer: A novel potential therapeutic target. Steroids 133: 102-107, 2018.

8. Huang JJ, Ma WJ and Yokoyama S: Expression and immunolocalization of Gpnmb, a glioma-associated glycoprotein, in normal and inflamed central nervous systems of adult rats. Brain Behav 2: 85-96, 2012.

9. Hu X, Zhang P, Xu Z, Chen H and Xie X: GPNMB enhances bone regeneration by promoting angiogenesis and osteogenesis: Potential role for tissue engineering bone. J Cell Biochem 114: 2729-2737, 2013.

10. Wang YL, Hu YJ and Zhang FH: Effects of GPNMB on proliferation and odontoblastic differentiation of human dental pulp cells. Int J Clin Exp Pathol 8: 6498-6504, 2015.

11. Ono $Y$, Tsuruma $K$, Takata $M$, Shimazawa $M$ and Hara $H$ : Glycoprotein nonmetastatic melanoma protein B extracellular fragment shows neuroprotective effects and activates the PI3K/Akt and MEK/ERK pathways via the $\mathrm{Na}+/ \mathrm{K}+$-ATPase. Sci Rep 6: 23241, 2016.

12. Nakano Y, Suzuki Y, Takagi T, Kitashoji A, Ono Y, Tsuruma K, Yoshimura S, Shimazawa M, Iwama T and Hara H: Glycoprotein nonmetastatic melanoma protein B (GPNMB) as a novel neuroprotective factor in cerebral ischemia-reperfusion injury. Neuroscience 277: 123-131, 2014.

13. Nagahara Y, Shimazawa M, Ohuchi K, Ito J, Takahashi H, Tsuruma K, Kakita A and Hara H: GPNMB ameliorates mutant TDP-43-induced motor neuron cell death. J Neurosci Res 95: 1647-1665, 2017.

14. Shi GD, Cheng X, Zhou XH, Fan BY, Ren YM, Lin W, Zhang XL, Liu S, Hao Y, Wei ZJ and Feng SQ: iTR AQ-based proteomics profiling of Schwann cells before and after peripheral nerve injury. Iran J Basic Med Sci 21: 832-841, 2018.

15. Kang W, Sun T, Tang D, Zhou J and Feng Q: Time-course transcriptome analysis of gingiva-derived mesenchymal stem cells reveals that fusobacterium nucleatum triggers oncogene expression in the process of cell differentiation. Front Cell Dev Biol 7: $359,2020$.

16. Ashburner M, Ball CA, Blake JA, Botstein D, Butler H, Cherry JM, Davis AP, Dolinski K, Dwight SS, Eppig JT, et al: Gene ontology: Tool for the unification of biology. The gene ontology consortium. Nat Genet 25: 25-29, 2000. 
17. The Gene Ontology Consortium: The gene ontology resource: 20 years and still GOing strong. Nucleic Acids Res 47 (D1): D330-D338, 2019.

18. Kanehisa M, Sato Y, Furumichi M, Morishima K and Tanabe M: New approach for understanding genome variations in KEGG. Nucleic Acids Res 47 (D1): D590-D595, 2019.

19. Zheng Y, Huang C, Liu F, Lin H, Niu Y, Yang X and Zhang Z: Reactivation of denervated Schwann cells by neurons induced from bone marrow-derived mesenchymal stem cells. Brain Res Bull 139: 211-223, 2018.

20. Livak KJ and Schmittgen TD: Analysis of relative gene expression data using real-time quantitative PCR and the 2(-Delta Delta $\mathrm{C}(\mathrm{T})$ ) method. Methods 25: 402-408, 2001

21. Ren T, Yu S, Mao Z and Gao C: A complementary density gradient of zwitterionic polymer brushes and NCAM peptides for selectively controlling directional migration of Schwann cells. Biomaterials 56: 58-67, 2015.

22. Weterman MA, Ajubi N, van Dinter IM, Degen WG, van Muijen GN, Ruitter DJ and Bloemers HP: nmb, a novel gene, is expressed in low-metastatic human melanoma cell lines and xenografts. Int J Cancer 60: 73-81, 1995.

23. Tanaka H, Shimazawa M, Kimura M, Takata M, Tsuruma K, Yamada M, Takahashi H, Hozumi I, Niwa J, Iguchi Y, et al: The potential of GPNMB as novel neuroprotective factor in amyotrophic lateral sclerosis. Sci Rep 2: 573, 2012.

24. Nagahara Y, Shimazawa M, Tanaka H, Ono Y, Noda Y, Ohuchi K, Tsuruma K, Katsuno M, Sobue G and Hara H: Glycoprotein nonmetastatic melanoma protein $\mathrm{B}$ ameliorates skeletal muscle lesions in a SOD1G93A mouse model of amyotrophic lateral sclerosis. J Neurosci Res 93: 1552-1566, 2015.

25. Gu X, Ding F, Yang Y and Liu J: Construction of tissue engineered nerve grafts and their application in peripheral nerve regeneration. Prog Neurobiol 93: 204-230, 2011.

26. Parrinello S, Napoli I, Ribeiro S, Wingfield Digby P, Fedorova M, Parkinson DB, Doddrell RD, Nakayama M, Adams RH and Lloyd AC: EphB signaling directs peripheral nerve regeneration through Sox2-dependent Schwann cell sorting. Cell 143: 145-155, 2010 .
27. Hall SM: The biology of chronically denervated Schwann cells. Ann N Y Acad Sci 883: 215-233, 1999.

28. Metz RL, Yehia G, Fernandes H, Donnelly RJ and Rameshwar P: Cloning and characterization of the 5 ' flanking region of the HGFIN gene indicate a cooperative role among p53 and cytokine-mediated transcription factors: Relevance to cell cycle regulation. Cell Cycle 4: 315-322, 2005.

29. Metz RL, Patel PS, Hameed M, Bryan M and Rameshwar P: Role of human HGFIN/nmb in breast cancer. Breast Cancer Res 9: R58, 2007.

30. Xu QG, Midha R, Martinez JA, Guo GF and Zochodne DW: Facilitated sprouting in a peripheral nerve injury. Neuroscience 152 : 877-887, 2008.

31. Li R, Li Y, Wu Y, Zhao Y, Chen H, Yuan Y, Xu K, Zhang H, Lu Y, Wang J, et al: Heparin-poloxamer thermosensitive hydrogel loaded with bFGF and NGF enhances peripheral nerve regeneration in diabetic rats. Biomaterials 168: 24-37, 2018.

32. Hei WH, Almansoori AA, Sung MA, Ju KW, Seo N, Lee SH, Kim BJ, Kim SM, Jahng JW, He H and Lee JH: Adenovirus vector-mediated ex vivo gene transfer of brain-derived neurotrophic factor (BDNF) tohuman umbilical cord blood-derived mesenchymal stem cells (UCB-MSCs) promotescrush-injured rat sciatic nerve regeneration. Neurosci Lett 643: 111-120, 2017.

33. Sahenk Z, Nagaraja HN, McCracken BS, King WM, Freimer ML, Cedarbaum JM and Mendell JR: NT-3 promotes nerve regeneration and sensory improvement in CMT1A mouse models and in patients. Neurology 65: 681-689, 2005.

34. Clements MP, Byrne E, Camarillo Guerrero LF, Cattin AL, Zakka L, Ashraf A, Burden JJ, Khadayate S, Lloyd AC, Marguerat S and Parrinello S: The wound microenvironment reprograms schwann cells to invasive mesenchymal-like cells to drive peripheral nerve regeneration. Neuron 96: 98-114.e7, 2017.

(i) (5) $\ominus$ This work is licensed under a Creative Commons Attribution-NonCommercial-NoDerivatives 4.0 International (CC BY-NC-ND 4.0) License. 